\title{
Size-biased discrete two parameter Poisson-Lindley Distribution for modeling and waiting survival times data
}

\author{
Tanka Raj Adhikari, R.S. Srivastava \\ Department of Statistics, Tribhuvan University, Prithvi Narayan Campus, Pokhara, Nepal \\ Deptartment of Mathematics and Statistics, DDU Gorakhpur University, Gorakhpur, India
}

\begin{abstract}
The main aim of this paper is to introduce and study the notion of Size-biased discrete two-parameter Poisson-Lindley (SBDTPPL) distribution. Besides deriving its p.m.f., some of its properties and the expressions for raw and central moments, coefficients of skewness and kurtosis have been obtained. We have explored its moment equations and maximum likelihood estimators. A simulation study has been proposed in the end.

Key Words: Size-biased Poisson-Lindley distribution, Compounding; raw and central moments; maximum likelihood estimators; Fisher's scoring method.
\end{abstract}

\section{Introduction}

Lindley (1958) has introduced a one-parameter distribution, known as Lindley distribution, given by probability density function (p.d.f.)

$$
f(x ; \theta)=\frac{\theta^{2}}{\theta+1}(1+x) e^{-\theta x} ; x>0, \quad \theta>0 . .
$$

where as one parameter Poisson-Lindley distribution (PLD) has been introduced by Sankaran (1970) to model count data with probability mass function (p.m.f.)

$$
f_{0}(x ; \theta)=\frac{\theta^{2}(x+\theta+2)}{(\theta+1)^{x+3}}, x=0,1, \ldots ; \theta>0 .
$$

The distribution arises from the Poisson distribution when its parameter $\lambda$ follows a Lindley distribution with probability density function (p.d.f.)

$$
\mathrm{g}_{0}(\lambda ; \theta)=\frac{\theta^{2}}{\theta+1}(1+\lambda) \mathrm{e}^{-\theta \lambda}, \quad \lambda>0, \quad \theta>0 .
$$

A Two-parameter Lindley distribution has been proposed in Shanker et al. (2013) of which one-parameter Lindley distribution (LD) is a particular case, for modeling waiting and survival time's data. Shanker and Mishra (2013) proposed a two-parameter Quasi Lindley Distribution (QLD) and studied its properties. In all the data-sets, it is found that the QLD provides closer fits than those by the Lindley distribution. Shanker et al. $(2012)^{2}$ proposed a discrete two parameter Poisson Lindley distribution (PLD), of which the Sankaran's Poisson-Lindley distribution (1970) is a special case. It is derived by compounding a Poisson distribution with the discrete two-parameter Lindley distribution of Shanker et al. $(2012)^{1}$. They derived first four moments of this distribution and have studied the estimation of the parameters by the method of moments. They have found that the two-parameter PLD is better fit and more flexible than the Shankaran'a one-parameter PLD to some data sets.

Ghitany and Al-Mutairi (2009) discussed some estimation methods for the discrete Poisson Lindley distribution (1.2) and its applications. They derived a discrete two-parameter Poisson Lindley distribution by compounding a Poisson distribution with a two-parameter Lindley distribution obtained by Shanker et al. $(2012)^{1}$.

In many a situation experimenters do not work with truly random sample from the population, in which they are interested, either by design or because of the fact that in many situations it becomes impossible to have random sample from the targeted population. However, since the observations do not have an equal probability of entering the sample, the resulting sampled distribution does not follow the original distribution. Statistical models that incorporate these restrictions are called weighted models. When an investigator records an observation by nature according to certain stochastic model, the recorded observation will not have the original distribution unless every observation is given an equal chance of being recorded. For example, suppose that the original observation $\mathrm{x}_{0}$ comes from a distribution with p.m.f./p.d.f. $\mathrm{f}_{0}\left(\mathrm{x}_{0}\right)$ and that observation $\mathrm{x}$ is recorded according to a probability re-weighted by a weight function $\mathrm{w}(\mathrm{x})>0$, then $\mathrm{x}$ comes from a distribution with p.m.f./p.d.f.

$$
f(x)=\frac{w(x)}{E\left[w\left(X_{0}\right)\right]} f_{0}(x) \ldots(1.4) \text {. }
$$


Rao (1965) introduced distributions of this type and called them weighted distributions. The weighted distribution with $\mathrm{w}(\mathrm{x})=\mathrm{x}$ is called size-biased/length-biased distribution. Patil and Rao (1978) examined some general models leading to weighted distributions and showed how the weight $\mathrm{w}(\mathrm{x})=\mathrm{x}$ occurs in a natural way in many sampling problems. A study of size-biased sampling and related form-invariant weighted distributions was made by Patil and Ord (1975). A survey of real-life applications of size-biased distributions may be found in Patil and Rao (1977, 1978).

Ghitany and Al-Mutairi (2008) proposed size-biased Poisson-Lindley distribution and suggested its applications. They consider the size-biased version of Poisson-Lindley distribution and obtained the p.m.f. of size-biased Poisson-Lindley (SBPL) distribution as

$$
f(x ; \theta)=\frac{x}{\mu_{0}} f_{0}(x ;)=\frac{\theta^{3}}{\theta+2} \frac{x(x+\theta+2)}{(\theta+1)^{x+2}}, \quad x=1,2, \ldots ; \theta>0, \ldots \text { (1.5) }
$$

where, $\mu_{0}=\frac{\theta+2}{\theta(\theta+1)}$ is the mean of the Poisson-Lindley distribution with p.m.f. (1.2).

The mean $\left(\mu_{1}\right)$, variance $\left(\mu_{2}\right)$, coefficient of skewness $\left(\sqrt{ } \beta_{1}\right)$ and coefficient of kurtosis $\left(\beta_{2}\right)$ for the SBPL distribution proposed by Ghitany and Al-Mutairi (2009) are as

$$
\begin{aligned}
\mu_{1} & =\frac{\theta^{2}+4 \theta+6}{\theta(\theta+2)}, \\
\mu_{2} & =\frac{2\left(\theta^{3}+6 \theta^{2}+12 \theta+6\right.}{\theta^{2}(\theta+1)^{2}}, \\
\sqrt{\beta_{1}} & =\frac{\theta^{5}+10 \theta^{4}+42 \theta^{3}+84 \theta^{2}+72 \theta+24}{\sqrt{2}\left(\theta^{3}+6 \theta^{2}+12 \theta+6\right)^{3 / 2}} ; \text { and } \\
\beta_{2} & =\frac{\theta^{7}+22 \theta^{6}+184 \theta^{5}+780 \theta^{4}+1800 \theta^{3}}{2\left(\theta^{3}+6 \theta^{2}+12 \theta+6\right)^{2}} .
\end{aligned}
$$

Adhikari and Srivastava (2013) proposed another size-biased Poisson-Lindley (SBPL) distribution obtained by compounding the size-biased Poisson distribution with Lindley distribution without considering its size-biased form. The size-biased Poisson distribution has the p.m.f.

$$
f(x / \lambda)=\frac{e^{-\lambda} \cdot \lambda^{x-1}}{(x-1) !} ; \quad x=1,2,3, \ldots, \quad \lambda>0, \ldots(1.6)
$$

Now if its parameter $\lambda$ follows the Lindley distribution with p.m.f. (1.1) then the p.m.f.of the size-biased Poisson-Lindley (SBPL) distribution is obtained as

$$
\begin{aligned}
f(x ; \theta)= & \int_{0}^{\infty} f(x / \lambda) \cdot g_{0}(\lambda ; \theta) d \lambda \\
& =\int_{0}^{\infty} \frac{e^{-\lambda} \cdot \lambda^{x-1}}{(x-1) !} \cdot \frac{\theta^{2}}{(\theta+1)} \cdot(1+\lambda) \cdot e^{-\theta \lambda} d \lambda \\
& =\frac{\theta^{2}}{(\theta+1)^{x+2}} \cdot(x+\theta+1), \quad x=1,2,3, \ldots(1.7),
\end{aligned}
$$

The mean $\left(\mu_{1}\right)$, variance $\left(\mu_{2}\right)$, coefficient of skewness $\left(\sqrt{ } \beta_{1}\right)$ and coefficient of kurtosis $\left(\beta_{2}\right)$ for another sizebiased Poisson Lindley (SBPL) distribution are as under

$$
\begin{aligned}
& \mu_{1}=\frac{\theta^{2}+2 \theta+2}{\theta(\theta+1)} \\
& \mu_{2}=\frac{\theta^{3}+4 \theta^{2}+6 \theta+2}{\theta^{2}(\theta+1)^{2}} \\
& \sqrt{\beta_{1}}=\frac{\theta^{5}+7 \theta^{4}+22 \theta^{3}+32 \theta^{2}+18 \theta+4}{\left(\theta^{3}+4 \theta^{2}+6 \theta+2\right)^{3 / 2}} ; \text { and } \\
& \beta_{2}=\frac{\mu_{4}}{\mu_{2}^{2}}=\frac{\theta^{7}+15 \theta^{6}+87 \theta^{5}+258 \theta^{4}+406 \theta^{3}+338 \theta^{2}+144 \theta+24}{\left(\theta^{3}+4 \theta^{2}+6 \theta+2\right)^{2}} .
\end{aligned}
$$

Adhikari and Srivastava (2014) proposed Poisson-size-biased Lindley (PSBL) distribution obtained by compounding the Poisson distribution without considering its size-biased form with size-biased Lindley distribution. The p.m.f. comes to be

$$
f(x ; \theta)=\int_{0}^{\infty} \frac{e^{-\lambda} \cdot \lambda^{x}}{x !} \cdot \frac{\theta^{3}}{(\theta+2)} \cdot \lambda(1+\lambda) \cdot e^{-\theta \lambda} d \lambda
$$




$$
=\frac{\theta^{3}}{(\theta+1)^{x+3}} \cdot \frac{1}{\theta+2} \cdot(x+1)(x+\theta+3), \quad x=0,1,2, \ldots
$$

Here the mean $\left(\mu_{1}\right)$, variance $\left(\mu_{2}\right)$, coefficient of skewness $\left(\sqrt{ } \beta_{1}\right)$ and coefficient of kurtosis $\left(\beta_{2}\right)$ of this distribution are as:

$$
\begin{gathered}
\mu_{1}=\frac{2 \theta^{3}+10 \theta^{2}+14 \theta+6}{\theta(\theta+2)(\theta+1)^{2}} \\
\mu_{2}=\frac{2 \theta^{7}+20 \theta^{6}+84 \theta^{5}+188 \theta^{4}+242 \theta^{3}+180 \theta^{2}+72 \theta+12}{\left\{\theta(\theta+2)(\theta+1)^{2}\right\}^{2}} \\
\gamma_{1}=\frac{2 \theta^{11}+32 \theta^{10}+234 \theta^{9}+1012 \theta^{8}+2842 \theta^{7}+5424 \theta^{6}+7190 \theta^{5}+6644 \theta^{4}+4212 \theta^{3}+1752 \theta^{2}+432 \theta+48}{\left(2 \theta^{7}+20 \theta^{6}+84 \theta^{5}+188 \theta^{4}+242 \theta^{3}+180 \theta^{2}+72 \theta+12\right)^{3 / 2}} \\
\gamma_{2}=\frac{2 \theta^{15}+48 \theta^{14}+860 \theta^{13}+12500 \theta^{12}+48728 \theta^{11}+176956 \theta^{10}+566162 \theta^{9}+2160028 \theta^{8}+882278 \theta^{7}+}{763716 \theta^{6}+492536 \theta^{5}+262695 \theta^{4}+107032 \theta^{3}+36480 \theta^{2}+3456 \theta+288} \\
\left(2 \theta^{7}+20 \theta^{6}+84 \theta^{5}+188 \theta^{4}+242 \theta^{3}+180 \theta^{2}+72 \theta+12\right)^{2}
\end{gathered}
$$

Shanker et al. (2012) proposed a two-parameter Lindley distribution given by the probability mass function (p.m.f.) as

$$
\mathrm{f}(\mathrm{x} ; \alpha, \theta)=\frac{\theta^{2}}{\theta+\alpha}(1+\alpha \mathrm{x}) \cdot \mathrm{e}^{-\theta \mathrm{x}}, \quad \mathrm{x}>0, \quad \theta>0, \quad \alpha>0 \ldots
$$

This distribution has been found to be a better model than the one-parameter Lindley distribution for analyzing waiting time, survival time and grouped mortality data. For $\alpha=1$, the distribution reduces to the one-parameter Lindley distribution (1.1). Suppose that the parameter $\lambda$ of a Poisson distribution follows the two parameter Lindley distribution (1.9), then the two-parameter Lindley mixture of Poisson distribution becomes

$$
\begin{aligned}
\mathrm{P}(\mathrm{x}) & =\int_{0}^{\infty} \frac{\mathrm{e}^{-\lambda} \cdot \lambda^{\mathrm{x}}}{\mathrm{x} !} \cdot \frac{\theta^{2}}{(\theta+\alpha)} \cdot(1+\alpha \lambda) \cdot \mathrm{e}^{-\theta \lambda} \mathrm{d} \lambda \\
& =\frac{\theta^{2}}{(\theta+1)^{\mathrm{x}+2}}\left[1+\frac{\alpha \mathrm{x}+\alpha}{\theta+\alpha}\right] ; \mathrm{x}=0,1,2, . . ; \theta>0 ; \alpha>0 \ldots
\end{aligned}
$$

This is called a discrete two-parameter Poisson-Lindley distribution. It can be seen that for $\alpha=1$, this distribution reduces to the one-parameter Poisson-Lindley distribution (1.2). For $\alpha=0$, it reduces to the geometric distribution with parameter, $\mathrm{p}=\frac{\theta}{\theta+1}$.

The first four raw moments of this distribution with p.m.f. (1.10) obtained by Shanker et al. (2012) are as

$$
\begin{aligned}
& \mu_{1}^{\prime}=\frac{\theta+2 \alpha}{\theta(\theta+\alpha)}, \quad \mu_{2}^{\prime}=\frac{\theta+2 \alpha}{\theta(\theta+\alpha)}+\frac{2(\theta+3 \alpha)}{\theta^{2}(\theta+\alpha)}, \\
& \mu_{3}^{\prime}=\frac{\theta+2 \alpha}{\theta(\theta+\alpha)}+\frac{6(\theta+3 \alpha)}{\theta^{2}(\theta+\alpha)}+\frac{6(\theta+4 \alpha)}{\theta^{3}(\theta+\alpha)} ; \text { and } \\
& \mu_{4}^{\prime}=\frac{\theta+2 \alpha}{\theta(\theta+\alpha)}+\frac{14(\theta+3 \alpha)}{\theta^{2}(\theta+\alpha)}+\frac{36(\theta+4 \alpha)}{\theta^{3}(\theta+\alpha)}+\frac{24(\theta+5 \alpha)}{\theta^{4}(\theta+\alpha)} .
\end{aligned}
$$

Moreover, for $\alpha=1$, these moments reduce to the respective moments of one-parameter Poisson-Lindley distribution with p.m.f. (1.2).

We also propose a discrete two-parameter Lindley-Size-biased Poisson (DTPLSBP) distribution by compounding the size-biased Poisson distribution with a discrete two-parameter Lindley distribution without considering its size-biased form (this paper is on the process of publication). The size-biased Poisson distribution has the p.m.f.

$$
f\left(\frac{x}{\lambda}\right)=\frac{e^{-\lambda} \cdot \lambda^{x-1}}{(x-1) !} ; \quad x=1,2,3, \ldots, \quad \lambda>0, \ldots
$$

Now if its parameter $\lambda$ follows the discrete two-parameter Lindley distribution with p.m.f. (1.9), then p.m.f. of a discrete two-parameter Lindley-Size-biased Poisson (DTPLSBP) distribution is obtained by using mixture model as:

$$
\begin{aligned}
f(x ; \alpha, \theta) & =\int_{0}^{\infty} g\left(\frac{x}{\lambda}\right) \cdot h(\lambda ; \theta) d \lambda \\
& =\int_{0}^{\infty} \frac{e^{-\lambda} \lambda^{x-1}}{(x-1) !} \cdot \frac{\theta^{2}}{\theta+\alpha} \cdot(1+\alpha \lambda) e^{-\theta \lambda} d \lambda
\end{aligned}
$$




$$
=\frac{\theta^{2}}{(\theta+1)^{\mathrm{x}+1}} \cdot \frac{1}{\theta+\alpha} \cdot(\theta+1+\alpha \mathrm{x}) \ldots
$$

Then the First four central moments, skewness, kurtosis and their coefficients of this Proposed a discrete twoparameter Lindley-size-biased Poisson (DTPLSBP) distribution with p.m.f. (1.12) are obtained as:

$$
\begin{gathered}
\mu_{1}=\frac{\theta(\theta+1)+\alpha(\theta+2)}{\theta(\theta+\alpha)} \\
\mu_{2}=\frac{2 \theta^{3}+\theta^{2}+3 \alpha \theta^{2}+4 \alpha \theta+2 \alpha^{2} \theta+2 \alpha^{2}}{\{\theta(\theta+\alpha)\}^{2}} \\
\mu_{4}=\frac{\theta^{5}+3 \theta^{4}+2 \theta^{3}+24 \theta^{2}+4 \alpha \theta^{4}+15 \alpha \theta^{3}-12 \alpha \theta^{2}+48 \alpha \theta+5 \alpha^{2} \theta^{3}+18 \alpha^{2} \theta^{2}-36 \alpha^{2} \theta+2 \alpha^{3} \theta^{2}+6 \alpha^{3} \theta-20 \alpha^{3}+24 \alpha^{2}}{\{\theta(\theta+\alpha)\}^{3}} \\
\theta^{7}+10 \theta^{6}+18 \theta^{5}+9 \theta^{4}+5 \alpha \theta^{6}+60 \alpha \theta^{5}+126 \alpha \theta^{4}+72 \alpha \theta^{3}+9 \alpha^{2} \theta^{5}+116 \alpha^{2} \theta^{4}+240 \alpha^{2} \theta^{3}+204 \alpha^{2} \theta^{2}+48 \alpha^{2} \theta \\
+7 \alpha^{3} \theta^{4}+92 \alpha^{3} \theta^{3}+132 \alpha^{3} \theta^{2}+192 \alpha^{3} \theta+2 \alpha^{4} \theta^{3}+2 \alpha^{4} \theta^{2}-96 \alpha^{4} \theta+144 \alpha^{3}-120 \alpha^{4}
\end{gathered}
$$

If we put $\alpha=1$, in the first four central moments, skewness, kurtosis and their coefficients of this proposed distribution then we get the corresponding one parameter a Size-biased Poisson-Lindley (SBPL) distribution with p.m.f. (1.7).

\section{Size-biased discrete two-parameter Poisson-Lindley distribution:}

In this paper we propose size-biased discrete two-parameter Poisson-Lindley (SBDTPPL) distribution by compounding the size-biased Poisson distribution with size-biased discrete two-parameter Lindley distribution with p.m.f.

$$
\begin{array}{r}
f(x ; \theta, \alpha)=\frac{x}{\mu_{0}} p(x)=\frac{\theta^{3}}{(\theta+1)^{x+2}} \cdot \frac{1}{\theta+2 \alpha} \cdot x(\theta+\alpha+1+\alpha x), \\
x=1,2, \ldots ; \theta>0, \quad \alpha>-\theta ; \ldots(2.1)
\end{array}
$$

where, $\mu_{0}=\frac{\theta+2 \alpha}{\theta(\theta+\alpha)}$ is the mean of a discrete two-parameter Poisson-Lindley distribution with p.m.f. (1.10). The Size-biased discrete two-parameter Poisson-Lindley distribution also arises from the size-biased Poisson (SBP) distribution with p.m.f.

$$
g(x / \lambda)=e^{-\lambda} \frac{\lambda^{x-1}}{(x-1) !}, \quad x=1,2, \ldots, \lambda>0, \ldots
$$

When its parameter $\lambda$ follows a size-biased discrete two-parameter Lindley model with p.d.f.

$$
\mathrm{h}(\lambda ; \theta, \alpha)=\frac{\theta^{3}}{\theta+2 \alpha} \lambda(1+\alpha \lambda) \mathrm{e}^{-\theta \lambda}, \quad \lambda>0, \theta>0, \ldots
$$

then the p.m.f. of this Size-biased discrete two-parameter Lindley distribution with the mixture of Poisson distribution is obtained by using mixture model as:

$$
\begin{aligned}
\mathrm{f}(\mathrm{x} ; \alpha, \theta) & =\int_{0}^{\infty} \mathrm{g}\left(\frac{\mathrm{x}}{\lambda}\right) \cdot \mathrm{h}(\lambda ; \theta) \mathrm{d} \lambda \\
& =\int_{0}^{\infty} \frac{e^{-\lambda} \lambda^{x-1}}{(x-1) !} \cdot \frac{\theta^{3}}{\theta+2 \alpha} \cdot \lambda(1+\alpha \lambda) e^{-\theta \lambda} d \lambda \\
& =\frac{\theta^{3}}{\theta+2 \alpha} \cdot \frac{1}{(x-1) !}\left[\int_{0}^{\infty} e^{-(\theta+1) \lambda} \lambda^{x} d \lambda+\int_{0}^{\infty} e^{-(\theta+1) \lambda} \alpha \cdot \lambda^{x+1} d \lambda\right] \\
& =\frac{\theta^{3}}{(\theta+1)^{x+2}} \cdot \frac{1}{\theta+2 \alpha} \cdot x(\theta+\alpha+1+\alpha x) \ldots(2.4)
\end{aligned}
$$

This is same as p.m.f. (2.1). The first four raw moments of this proposed size-biased discrete two-parameter Poisson-Lindley (SBDTPPL) distribution with p.m.f. (2.4) comes out to be

$$
\begin{aligned}
& \mu_{1}^{\prime}=\frac{\theta^{3}+3 \theta^{2}+2 \alpha \theta^{2}+8 \alpha \theta+2 \theta+6 \alpha}{\theta(\theta+1)(\theta+2 \alpha)}, \\
& \mu_{2}^{\prime}=\frac{\theta^{4}+7 \theta^{3}+12 \theta^{2}+6 \theta+2 \alpha \theta^{3}+20 \alpha \theta^{2}+42 \alpha \theta+24 \alpha}{\theta^{2}(\theta+1)(\theta+2 \alpha)}, \theta^{3}(\theta+1)(\theta+2 \alpha) \\
& \mu_{3}^{\prime}=\frac{\theta^{5}+15 \theta^{4}+50 \theta^{3}+60 \theta^{2}+24 \theta+2 \alpha \theta^{4}+44 \alpha \theta^{3}+186 \alpha \theta^{2}+264 \alpha \theta+120 \alpha}{\theta^{4}(\theta+1)(\theta+2 \alpha)} \\
&=\frac{\theta_{4}^{\prime}+31 \theta^{5}+180 \theta^{4}+390 \theta^{3}+360 \theta^{2}+120 \theta+2 \alpha \theta^{5}+92 \alpha \theta^{4}+690 \alpha \theta^{3}+1800 \alpha \theta^{2}+1920 \alpha \theta+720 \alpha}{}
\end{aligned}
$$


The mean $\left(\mu_{1}\right)$ and variance $\left(\mu_{2}\right)$ are obtained as:

$$
\begin{gathered}
\mu_{1}=\frac{\theta^{3}+3 \theta^{2}+2 \alpha \theta^{2}+8 \alpha \theta+2 \theta+6 \alpha}{\theta(\theta+1)(\theta+2 \alpha)}, \\
\mu_{2}=\frac{2 \theta^{5}+6 \theta^{4}+6 \theta^{3}+2 \theta^{2}+10 \alpha \theta^{4}+32 \alpha \theta^{3}+34 \alpha \theta^{2}+12 \alpha \theta+12 \alpha^{2} \theta^{3}+36 \alpha^{2} \theta^{2}+36 \alpha^{2} \theta+12 \alpha^{2}}{\{\theta(\theta+1)(\theta+2 \alpha)\}^{2}}
\end{gathered}
$$

If $\alpha=1$, then we get the corresponding mean and variance of the Size-biased one-parameter Poisson-Lindley distribution.

We now give some basic properties of the SBDTPPL model.

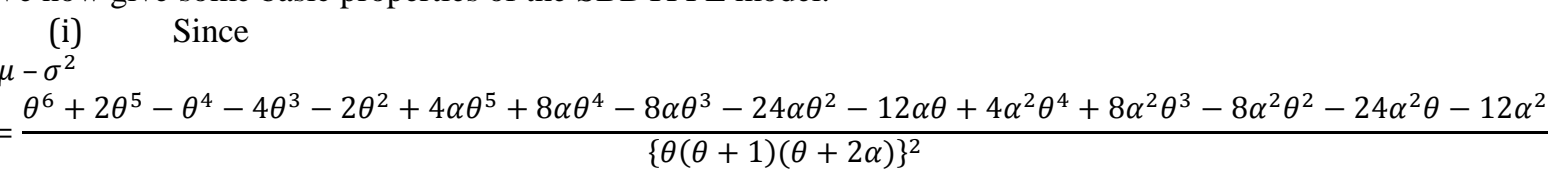

(ii) Since

$$
\frac{f(x+1 ; \theta)}{f(x ; \theta)}=\left(\frac{1}{\theta+1}\right) \cdot\left(1+\frac{1}{x}\right) \cdot\left(1+\frac{\alpha}{\theta+\alpha+1+\alpha x}\right)
$$

Is a decreasing function in $\mathrm{x}, \mathrm{f}(\mathrm{x} ; \theta)$ is log-concave. Therefore the SBDTPPL distribution is unimodal, has an increasing failure rate ( IFR ) and hence, increasing failure rate average (IFRA), new better than used (NBU), new better than used in expectation (NBUE) and decreasing mean residual life (DMRL) in Barlow and Proschan (1981) for more details about the definition of these aging concepts are given.

\section{Method of Moments}

Given a random sample $\mathrm{x}_{1}, \mathrm{x}_{2}, \mathrm{x}_{3}, \ldots, \mathrm{x}_{\mathrm{n}}$, of size $\mathrm{n}$ from the SBPL distribution with p.m.f.(2.2), the MOM estimate, $\tilde{\theta}$ of $\theta$ is given by

$$
\begin{aligned}
& E(X)=\frac{\theta^{3}+3 \theta^{2}+2 \alpha \theta^{2}+8 \alpha \theta+2 \theta+6 \alpha}{\theta(\theta+1)(\theta+2 \alpha)}=\bar{x} \\
& \text { Or, } \theta^{3}+3 \theta^{2}+2 \alpha \theta^{2}+8 \alpha \theta+2 \theta+6 \alpha-\bar{x} \theta^{3}-\bar{x} \theta^{2}-2 \alpha \bar{x} \theta^{2}-2 \alpha \bar{x} \theta=0 \\
& \text { Or, }(1-\bar{x}) \theta^{3}+(3+2 \alpha-2 \alpha \bar{x}-\bar{x}) \theta^{2}+(2+8 \alpha-2 \alpha \bar{x}) \theta+6 \alpha=0
\end{aligned}
$$

Note that $\bar{x}=1$ if and only if $\mathrm{x}_{\mathrm{i}}=1$ for all $\mathrm{i}=1,2 \ldots \mathrm{n}$. A data set where all observations are ones is not worth analyzing. This situation, o course, will not lead to any estimate of $\theta$. However, such situation may arise in a simulation experiment when $\mathrm{n}$ is small. For this reason, we will assume throughout this paper that $\bar{x}>1$.

\section{Maximum Likelihood:}

Given a random sample $\mathrm{x}_{1}, \mathrm{x}_{2}, \ldots, \mathrm{x}_{\mathrm{n}}$ of size $\mathrm{n}$ from the SBDTPPL distribution with p.m.f. (2.2) is,

$$
f(x ; \theta, \alpha)=\frac{\theta^{3}}{(\theta+1)^{x+2}} \cdot \frac{1}{\theta+2 \alpha} \cdot x(\theta+\alpha+1+\alpha x) ; \quad x=1,2, \ldots, \theta>0 \alpha>0
$$

The likelihood function will be

Taking log both sides

$$
\begin{aligned}
L\left(x_{i} ; \theta, \alpha\right) & =\prod_{i=1}^{n} f\left(x_{i} ; \theta, \alpha\right) \\
& =\left(\frac{\theta^{3}}{(\theta+1)^{2}} \cdot \frac{1}{\theta+2 \alpha}\right)^{n} \cdot \prod_{i=1}^{n} \frac{1}{(\theta+1)^{x_{i}}} \cdot x_{i}\left(\theta+\alpha+1+\alpha x_{i}\right)
\end{aligned}
$$

$$
\begin{aligned}
\log L= & 3 n \log \theta-2 n \log (\theta+1)-n \log (\theta+2 \alpha) \\
& +\sum_{i=1}^{n} \log x_{i}+\sum_{i=1}^{n} \log \left(\theta+\alpha+1+\alpha x_{i}\right)-\sum_{i=1}^{n} x_{i} \log (\theta+1) \\
\frac{\partial \log L}{\partial \alpha}= & \frac{-2 n}{\theta+2 \alpha}+\sum_{i=1}^{n} \frac{1+x_{i}}{\left(\theta+\alpha+1+\alpha x_{i}\right)} \ldots(3.1)_{a} \\
\frac{\partial \log L}{\partial \theta}= & \frac{3 n}{\theta}-\frac{2 n}{\theta+1}-\frac{n \bar{X}}{\theta+1}-\frac{n}{\theta+2 \alpha}+\sum_{i=1}^{n} \frac{1}{\left(\theta+\alpha+1+\alpha x_{i}\right)}
\end{aligned}
$$


$O R$

$$
\frac{\partial \log L}{\partial \theta}=\frac{3 n}{\theta}-\frac{n(2+\bar{X})}{\theta+1}-\frac{n}{\theta+2 \alpha}+\sum_{i=1}^{n} \frac{1}{\left(\theta+\alpha+1+\alpha x_{i}\right)} \ldots
$$

Thus the ML estimate $\hat{\theta}$ of $\theta$ is the solution of the non-linear equation

$$
\frac{3 n}{\theta}-\frac{n(2+\bar{x})}{\theta+1}-\frac{n}{\theta+2 \alpha}+\sum_{i=1}^{n} \frac{1}{\theta+\alpha+1+\alpha x_{i}}=0
$$

The two equations (3.1) $)_{a}$ and (3.1) $)_{b}$ may be solved by appropriate numerical methods. However, the Fisher Scoring Method can be applied to solve these equations. For, we have

$$
\begin{aligned}
& \frac{\partial^{2} \log L}{\partial \theta^{2}}=-\frac{3 \mathrm{n}}{\theta^{2}}+\frac{\mathrm{n}(2+\overline{\mathrm{X}})}{(\theta+1)^{2}}+\frac{\mathrm{n}}{(\theta+2 \alpha)^{2}}-\sum_{\mathrm{i}=1}^{\mathrm{n}} \frac{1}{\left(\theta+\alpha+1+\alpha \mathrm{x}_{\mathrm{i}}\right)^{2}} \ldots \\
& \frac{\partial^{2} \log \mathrm{L}}{\partial \alpha^{2}}=\frac{4 \mathrm{n}}{(\theta+2 \alpha)^{2}}-\sum_{\mathrm{i}=1}^{\mathrm{n}}\left(\frac{1+\mathrm{x}_{\mathrm{i}}}{\theta+\alpha+1+\alpha \mathrm{x}_{\mathrm{i}}}\right)^{2} \ldots(3.3)_{\mathrm{b}} \\
& \frac{\partial^{2} \log \mathrm{L}}{\partial \alpha \partial \theta}=\frac{2 \mathrm{n}}{(\theta+2 \alpha)^{2}}-\sum_{\mathrm{i}=1}^{\mathrm{n}} \frac{1+\mathrm{x}_{\mathrm{i}}}{\left(\theta+\alpha+1+\alpha \mathrm{x}_{\mathrm{i}}\right)^{2}} \ldots(3.3)_{\mathrm{c}}
\end{aligned}
$$

The following equations for $\hat{\theta}$ and $\hat{\alpha}$ can be solved

$$
\left[\begin{array}{ll}
\frac{\partial^{2} \log L}{\partial \theta^{2}} & \frac{\partial^{2} \log L}{\partial \alpha \partial \theta} \\
\frac{\partial^{2} \log L}{\partial \alpha \partial \theta} & \frac{\partial^{2} \log L}{\partial \alpha^{2}}
\end{array}\right]_{\substack{\hat{\theta}=\theta_{0} \\
\hat{\alpha}=\alpha_{0}}}\left[\begin{array}{l}
\hat{\theta}-\theta_{0} \\
\hat{\alpha}-\alpha_{0}
\end{array}\right]=\left[\begin{array}{c}
\frac{\partial \log L}{\partial \theta} \\
\frac{\partial \log L}{\partial \alpha}
\end{array}\right]_{\substack{\hat{\theta}=\theta_{0} \\
\hat{\alpha}=\alpha_{0}}}
$$

Where $\theta_{0}$ and $\alpha_{0}$ are the initial values of $\theta$ and $\alpha$ respectively. These equations are solved iteratively till sufficiently closed estimates of $\widehat{\theta}$ and $\widehat{\alpha}$ are obtained.

\section{References:}

[1]. Adhikari, T.R. and Srivastava, R.S. (2013): A Size-biased Poisson-Lindley Distribution, International Journal of Mathematical Modeling and Physical Sciences, Vol. 01 (3), pp. 1-5.

[2]. Adhikari, T.R. and Srivastava, R.S. (2014): Poisson-Size-biased Lindley Distribution,International Journal of Scientific and Research Publication, Vol. 4 (3), pp. 1-6.

[3]. Bakouch, H.S., Al-Zahrani, B.M., Al-Shomrani, A.A., Marchi, A.A. and Louzada, F. (2012) An Extended Lindley Distribution. Journal of the Korean Statistical Society, Vol. 41, No 1, pp. 75-85.

[4]. Barlow, R.E. and Proschan, F. (1981): Statistical Theory of Reliability and Life Testing, Silver Spring, MD: To Begin with.

[5]. Beall, G. (1940). The fit and significance of contagious distributions when applied to observations on larva insects. Ecology 21, 460-474.

[6]. Bjerkedal, T. ((1960) Acquisition of Resistence in Guinea Pigs Infected with Different Doses of Virulent Tubercle Ba-cilli. American Journal of Epidemiology, Vol. 72, No. 1, pp. 130-148.

[7]. Borah, M. (1984). The Genenbauer distribution revisited: Some recurrence relation for moments, cumulants, etc., estimation of parameters and its goodness of fit. Journal of Indian Society of Agricultural Statistics 36, 72-78.

[8]. Deniz, E.G. and Ojeda, E.C. (2011). The Discrete Lindley Distribution-Properties and Applications. Journal of Statistical Computation and Simulation, Vol. 81, No. 11, pp. 1405-1416.

[9]. Gentle, J.E. (2003) Random Number generation and Monte Carlo Methods, New York: Springer-Verlag, Second edition.

[10]. Ghitany, M.E., Atieh, B. and Nadarajah, S. (2008) "Lindley Distribution and its Applications," Mathematics and Computers in Simulation, Vol. 78, No. 4, pp. 493-506.

[11]. Ghitnany, M.E. and Al-Mutairi, D.K. (2008): Size-biased Poisson-Lindley Distribution and its Application: METRON International Journal of Statistics, Vol. LXVI, n-3, pp. 299-31.

[12]. Ghitany, M.E. and Al-Mutairi, D.K. (2009): Estimation Methods for the discrete Poisson-Lindley distribution. Journal of Statistical Computation and Simulation 79(1), 1-9.

[13]. Ghitany, M.E., Alqallaf, F., Al-Mutairi, D.K. and Hussain, H.A. A two Parameter Weighted Lindley Distribution and its Applications to Survival Data. Mathematics and Computers in Simulation, Vol. 81, No. 6, pp. 1190-1201.

[14]. Hogg, R.V., McKean, J.W. and Craig, A.T. (2005) Introduction to Mathematical Statistics, New Jersey: Pearson Prentice Hall, Sixth edition.

[15]. Kemp, C.D. and Kemp, A.W. (1965). Some properties of the Hermite distribution, Biometrika 52, 381-394.

[16]. Lindley, D.V. (1958): Fiducial Distribution and Bayes Theorem. Journal of Royal Statistical Society, Series B, 20, No.1, 102-107.

[17]. Majucheli, J. and Achcar, J.A. (2011) The Lindley distribution applied to Competing Risks Lifetime Data. Computer Methods and Programs in Biomedicine, Vol. 104, No. 2. Pp. 188-192.

[18]. Paranjpe, S. and Rajarshi, M.B. (1986) Modeling Non-Monotonic Survivorship Data with Bath Tube Distributions. Ecology, Vol. 67, No. 6, pp. 1693-1695.

[19]. Patil, G.P. and Ord, J.K. (1975): On size-biased sampling and related form-invariant weighted distributions, Sankhya, 38, 48-61.

[20]. Patil, G.P. and Rao, C.R. (1977). Weighted distributions: a survey of their applications, in: P.R. Krishnaiah (Ed.), Applications of Statistics Amsterdam, North-Holand, 383-405.

[21]. Patil, G.P. and Rao, C.R. (1978). Weighted distributions and size-biased sampling with applications to wildlife populations and human families, Biometrics, 34, 179-189.

[22]. Rao, CR. (1965). On discrete distributions arising out of ascertainment, In: Classical and Contagious discrete distribution; G.P. Patil (ed.), Pergamon press and Statistical Publishing Society, Calcutta, 302-332. 
[23]. Shankaran, M.(1970). The discrete Poisson-Lindley distribution. Biometric Vol. 26,No. 1, pp. 145-149.

[24]. Shanker, R., Sharma, S. and Shanker, R. (2012) ${ }^{1}$ A two-parameter Lindley Distribution for modeling waiting and survival times data. (Accepted for publication in Applied Mathematics).

[25]. Shanker, R., Sharma, S. and Shanker, R. (2012) $)^{2}$ A discrete two-parameter Poisson Lindley Distribution: JESA, Vol. XXI, pp. 1522.

[26]. Shanker, R., Sharma, S. and Shanker, R. (2013) A two-parameter Lindley Distribution for Modeling Waiting and Survival Times Data; doi: 10.4236/am. 2013, 42056 Publishd Online Februry 2013 (http://www.scirp.org/journal/am) Applied Mathematics, 2013, 4 363-368.

[27]. Shanker, R. and Mishra, A. (2013) A Quasi Lindley Distribution: African Journals of Mathematics and Computer Science Research, Vol. 6(4), pp. 64-71. 